\title{
Development of Fatal Bilateral Xanthogranulomatous Pyelonephritis in a Paraplegic Patient: Case Report
}

\author{
A. K. Goswami, MS, MCh(Urol), B. Suryaprakash, MS, MCh(Urol), \\ A. K. Malik, MD(Path), S. Vaidyanathan, MS, MCh(Urol), MNAMS, \\ PhD \\ Departments of Urology and Pathology, Postgraduate Institute of Medical Edu- \\ cation and Research, Chandigarh 160012 India
}

\begin{abstract}
Summary
The first case of a bilateral xanthogranulomatous pyelonephritis (XGPN) is reported in a paraplegic patient who did not practise intermittent catheterisation after discharge from the hospital but resorted to the Crede manoeuvre which predisposed to a fatal urinary tract infection and development of XGPN over a period of 5 weeks. The advantages of clean, unsterile, intermittent self catheterisation over the Crede manoeuvre in patients with a neuropathic bladder are discussed especially in preventing infective complications of the upper urinary tract which may prove life threatening as occurred in this patient.
\end{abstract}

Key words: Paraplegia; Catheterisation; Xanthogranulomatous pyelonephritis; Urinary tract infections.

Clean, unsterile, intermittent self-catheterisation was popularised by Lapides (Lapides et al., 1974) in patients with a neuropathic bladder. However, this concept has not found favour with family physicians and patients especially in their home environment. The Crede manoeuvre appears to be innocuous to them, but may endanger the patient. Such an example of a changeover from intermittent catheterisation to the Crede manoeuvre, based on the advice of a community family physician resulting in the development of pyonephrosis, renal failure and fatal septicemia is presented. Interestingly, autopsy revealed bilateral xanthogranulometous pyelonephritis (XGPN) which probably represents the first case of bilateral XPGN in a spinal cord injured person.

\section{Case report}

B.S., 25, M. farmer, fell from a height of 15 feet as a result of receiving an electric shock while performing repair work at his tube-well. Within 24 hours of 
the injury he was admitted to an emergency medical service on June 20, 1983 with traumatic paraplegia due to fracture of $\mathrm{L}_{1}$ vertebra. For his neuropathic bladder problem, clean unsterile intermittent catheterisation (I.C.) every 4 hours was advised, initially by the attendants and subsequently by the patient himself. When the patient was discharged on July 22, 1983, his urine culture was sterile, the blood urea was $30 \mathrm{mg}_{\%}^{\circ}$ and the serum creatinine was $1.6 \mathrm{mg} \%$. Excretory urography, before discharge, showed normal upper urinary tracts. He was advised to continue I.C. at 4 hourly intervals at home and to return for followup, after a month. Unfortunately, at home, the patient discontinued I.C. and was glad to accept the Crede manoeuvre on the advice of a family physician which helped him to avoid catheterisation and genital manipulation in a setting of a joint rural family where adequate privacy was not available. About 5 weeks later, on August 25, 1983, he was brough to the hospital with a high fever $\left(40^{\circ} \mathrm{C}\right)$ bilateral flank masses, palpable bladder, anaemia and azotemia. (Blood urea $300 \mathrm{mg}$ and Serum creatinine $8 \mathrm{mg} \%$ ). A 16 Fr. Foley catheter could be passed easily and $600 \mathrm{ml}$ of purulent urine was drained. Plain X-ray KUB revealed no calculi; an infusion pyelogram revealed non-visualisation of both kidneys and a cystogram showed a large capacity bladder with no V.U. reflux. Urine culture yielded E.Coli. Blood culture report received posthumously also showed E.Coli. In spite of antibiotics and other supportive measures, the patient died 48 hours after admission because of uncontrolled septicemia.

\section{Pathology}

At autopsy, each kidney weighed 650 gms. The surface was pale and capsule stripped off easily. On section the corticomedullary distinction was lost and there were multiple small abscesses with thin pus oozing out of them (Fig. 1). Histologically both kidneys showed similar features. The pathology was restricted to the tubules and interstitium. The glomeruli were normal. There were small scattered abscesses consisting of polymorphs interspersed with large collections of histiocytes (Fig. 2). These histocytes were large with abundant deep eosinophilic cystoplasm and are characteristic of XGPN (Fig. 3). Differential diagnosis of malakoplakia could not be substantiated as MichaelisGutmann bodies were neither seen on routine H\&E stain, nor on special stains like Perl's and periodic acid schiff (PAS) stains. Lungs, liver and spleen showed features of septicemia.

\section{Discussion}

Preservation of the normal upper urinary tract and prevention of urinary infection are the paramount treatment goals in the management of patients with a neuropathic bladder. Urinary tract infection is probably the most common complication in such patients (Slade, 1977). Neurogenic vesical dysfunction especially in the presence of high residual urine predisposes to infection. The defence mechanisms of the lower urinary tract are diminished in an overdistended bladder. Good hydration, frequent emptying and low residual urine will improve the blood supply and the defence mechanisms of the lower urinary tract. The programme of I.C. is an effective policy to achieve these objectives. 


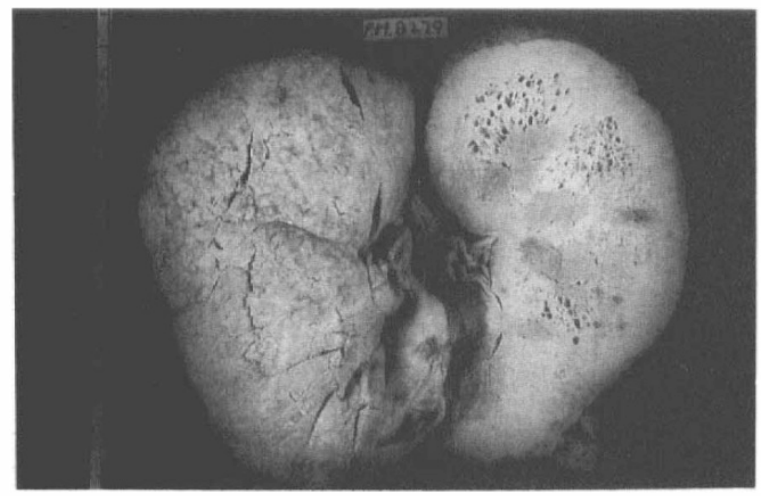

Figure 1 Macrograph of kidneys, outer and cut surfaces studded with small abscesses.

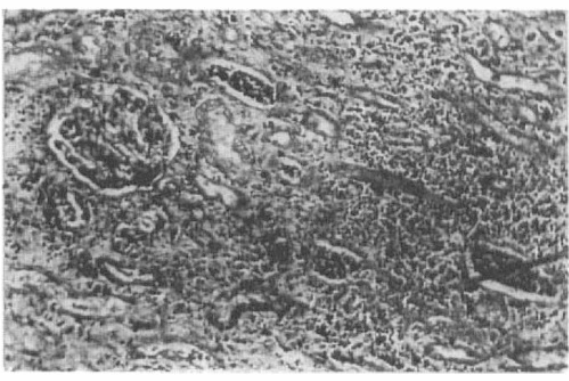

Figure 2 Micrograph of kidney showing normal glomeruli acute interstitial inflammation, tubular atrophy and purulent casts in the tubules. $\mathrm{H} \& \mathrm{E} \times 140$.

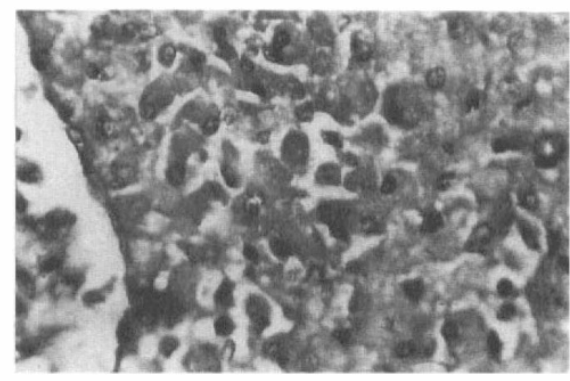

Figure 3 Micrograph showing collection of large histiocytes. H\&E X 550 .

Abdominal straining and the Crede manoeuvre in patients with suprasacral lesions will elicit a reflex contraction of the pelvic floor musculature and a consequent increase in the obstruction (Raj et al., 1979). Failure to maintain a low residual urine leads to back pressure changes and infection, as happened in this case. The upper urinary tract infection usually results in pyelonephritis, pyonephrosis, paranephric abscess or multiple cortical abscesses, but very rarely it takes the special form of xanthogranulomatous inflammatory lesion of the kidney (xanthogranulomatous pyelonephritis).

XGPN is a rare inflammatory condition of the kidney. Bilateral XGPN is rarer still. Meares could not find any case of such bilateral disease in the literature (Meares, 1978). However, Rossi, Vandenris and Smith reported one case each of bilateral XGPN (Rossi et al., 1968, Vandenris et al., 1976 and Smith, 1981). All these three cases of bilateral XGPN were described in patients without neurogenic vesical dysfunction. In the spinal cord injured unilateral XGPN has been reported in one paraplegic $\left(T_{6}\right)$ by Hooper (Hooper et al., 1962) and in 3 paraplegics $\left(\mathrm{L}_{3}, \mathrm{~T}_{11}\right.$ and $\mathrm{T}_{5}$ ) by Kawaichi (Kawaichi et al., 1967). Our patient probably represents the first example of bilateral XGPN in a paraplegic patient. 


\section{References}

HOOPER RG, KEMPSON RL, SCHLEGEL JU 1962 Xanthogranulomatous pyelonephritis. Fournal of Urology 88:585-593.

KAWAICH GK, REINGOLD IM 1967 Xanthogranulomatous pyelonephritis in the paraplegic. Fournal of Urology 97:58-61.

LAPIDES J, DIOKNO AC, LOWE BS, KALISH MO 1974 Follow-up on unsterile intermittent self catheterization. Fournal of Urology 111:184-187.

MEARES EM 1978 Urinary tract infection in men. Cambell's Urology, W. B. Saunders Company, Philadelphia. 513-514.

RAJ S, BRADLEY WE 1978 Neuromuscular dysfunction of the lower urinary tract. Cambell's Urology, W. B. Saunders Company, Philadelphia. 1215-1265.

Rossi P, MYers DH, FUREY R, BONFILS-ROBERTS EA 1968 Angiography in bilateral xanthogranulomatous pyelonephritis. Radiology 90:320-321.

SLADE N 1977 Urinary tract infections in neurogenic bladder. Fournal of Antimicrobilogy and Chemotherapy 3:3-5.

SMITH FR 1981 Bilateral xanthogranulomatous pyelonephritis. British fournal of Urology 53:81.

VANDENRIS M, Struyven J, Mathieu J, Schulman CC 1976 Bilateral xanthogranulomatous pyelonephritis. F. de Radiologie, D'Electroglogic et de Medicine Nucleaire 57:891-893. 